\title{
Study on the Design of a High Condensing LED Searchlight
}

\author{
Tae-Seong Kim, Jin-Wook Kim, Sun-Jae Kim, and Gyung-Suk Kil ${ }^{\dagger}$ \\ Dept. of Electrical and Electronics Engineering, Korea Maritime and Ocean University, Busan 606-791, Korea
}

Received June 8, 2015; Revised July 29, 2015; Accepted August 5, 2015

\begin{abstract}
This paper dealt with the condensing technology of an LED light source that uses a parabolic reflector to replace a searchlight equipped with a xenon lamp. A ray-tracing simulation was conducted to analyze the influence of the diameter of the reflector and the size of the light source on light condensing. The combination of a parabolic reflector with a diameter of $620 \mathrm{~mm}$ and a focal distance of $220 \mathrm{~mm}$, and a $9 \mathrm{~mm}$ multi-chip package (MCP) with a luminous flux of 7,000 lm showed the narrowest beam angle. The luminous intensity at the center was measured at $7.7 \times 10^{6} \mathrm{~cd}$. The distance between the light source and the point where the illuminance was $1 \mathrm{~lx}$ was calculated to be $2.8 \mathrm{~km}$. The power consumption of the system was $95 \mathrm{~W}$, which is only $9.5 \%$ of that of the $1 \mathrm{~kW}$ xenon searchlight, and the beam angle was $1.03^{\circ}$. In a site experiment, it was confirmed that the light ray reflected from the LED searchlight proceeds forward without any diffusion because of the narrow beam angle.
\end{abstract}

Keywords: LED searchlight, Light condensing, Luminous intensity, Beam angle, Parabolic reflector

\section{INTRODUCTION}

Light emitting diode (LED) has been regarded as a light source with a higher efficiency and longer lifetime than the conventional incandescent and discharge lamps such as fluorescent lamps and metal halide lamps. Widespread applications have been found for LEDs in the lighting industry within a short period of time. The light efficacy of an LED is over $80 \%$, while that of an incandescent lamp and a fluorescent lamp are $5 \%$ and $40 \%$, respectively [1,2]. The size of the domestic market for LEDs is estimated to increase to 17.7 trillion won in 2018 , with an average growth rate of $21 \%$ per year, from 2.1 trillion won in 2010 . When the conventional light sources with a domestic market share of $30 \%$ are replaced by LEDs, the power consumption will be reduced by 16,021 GWh per year, amounting to savings of about 1.6 trillion won. This is consistent with the global environmental regulation policy to reduce carbon dioxide emission by 6.8 mil-

${ }^{\dagger}$ Author to whom all correspondence should be addressed:

E-mail: kilgs@kmou.ac.kr

Copyright $(02015$ KIEEME. All rights reserved. This is an open-access article distributed under the terms of the Creative Commons Attribution Non-Commercial
License (http:///creativecommmons.org/licenses/by-nc/3.0) which permits unrestricted noncommercial use.

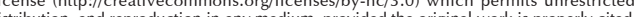

lion tons [3]. A xenon lamp of between $1 \mathrm{~kW}$ and $3 \mathrm{~kW}$ is currently used as a high-power searchlight on land or on ships in the sea. However, its inherent purpose is difficult to meet because its light source life is quite short - 350 hours, and it takes about ten minutes for full-lighting and restart.

In this paper, the design method of a high condensing LED searchlight with a parabolic reflector is proposed as a basic study for replacing the xenon searchlights used in coastguard stations and ships.

\section{DESIGN AND FABRICATION}

\subsection{Ray-tracing}

A parabolic reflector has been used to collect light or radio waves at a point in the solar concentrator and radio antenna [4]. The concept of condensation is shown in Fig. 1. If the function of a parabolic reflector is reversed, the light emitting from the point will be reflected in parallel, and the beam will acquire the maximum luminous intensity at the center of the LED searchlight $[5,6]$. 


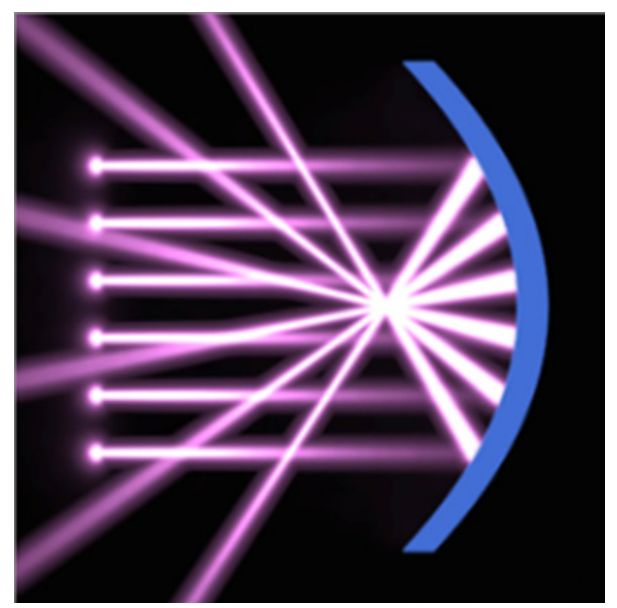

Fig. 1. Condensing concept of a parabolic reflector.

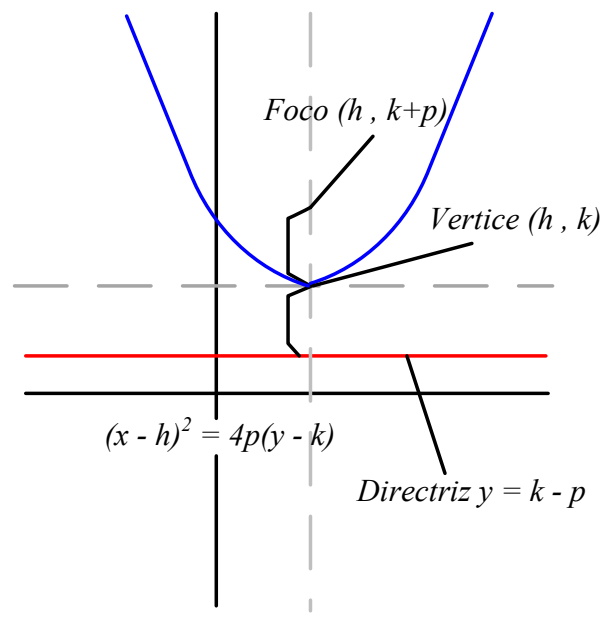

Fig. 2. A parabolic reflection and a focal distance.

The parabolic reflection and focal distance are shown in Fig. 2 and defined by equation (1), where $x$ is the outer radius, $y$ is the depth and $p$ is the focal distance. As the outer radius increases and the depth reduces, the focal distance increases.

$$
\begin{aligned}
& (x-h)^{2}=4 p(y-k) \\
& x^{2}=4 p y \\
& P \propto \frac{1}{y}, P \propto x^{2}
\end{aligned}
$$

The optimal focal point is proposed by ray-tracing simulation as shown in Fig. 3(a), since an LED as a surface light source is depart from the focus of the parabolic reflector, while a point light source is satisfied with the function of a parabolic reflector as shown in Fig. 2 [7].

Point $\mathrm{A}$ is the ray coming above the focal point into the top of the parabolic reflector, point $\mathrm{B}$ is the ray coming in line with the focal point into the parabolic reflector, and point $\mathrm{C}$ is the ray coming below the focal point into the bottom of the parabolic reflector. (a)
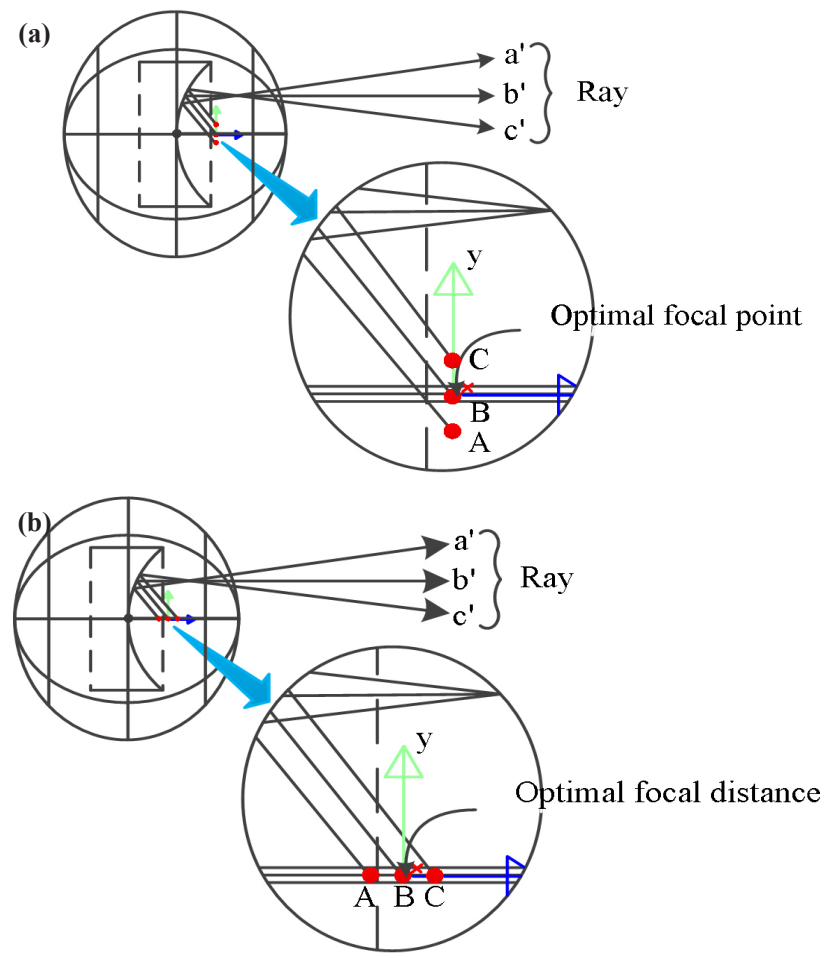

Fig. 3. Reflection against ray direction. (a) vertical line and (b) horizontal line.

Ray b' is parallel with the horizontal line from the center of the parabolic reflector, while rays a' and c', above and below the focus respectively, are diffused or focused at a point on the parabolic reflector [8]. The simulation result shows that the diameter and the size of the light source should be reduced to reflect the rays in parallel.

Figure 3(b) shows the ray direction in accordance with the focal distance from the $\mathrm{x}$-axis. Ray a', which is emitted from the point closer to the reflector is diffused and ray c', which is emitted from a point some distance from the reflector, is focused at a point. Since rays a' and c' are not suitable for searchlights, ray b', which is parallel, should be reflected by changing the focal distance.

\subsection{Calculation of luminous intensity}

The performance of the searchlight is evaluated using the luminous intensity at the center. Luminous intensity is defined as the magnitude of luminous flux emitted inside a solid angle from a light source in a particular direction, as shown in Fig. 4. It is expressed in cd [candela]. In this paper, the luminous intensity was calculated with luminance, luminous flux, and solid angle to evaluate the performance of the searchlight.

Luminous flux is the measure of the perceived power of light in the range of $380 \mathrm{~nm} \sim 780 \mathrm{~nm}$, and is expressed in lumen. Therefore, in the case of other light sources except for the point sources, luminous intensity could be calculated using equation (4). Where $\Phi$ is the luminous flux, $I$ is the luminous intensity, and $\omega$ is the solid angle.

$$
\phi=I \bullet \omega[\operatorname{lm}]
$$

The solid angle $\omega$ of a point light source is $4 \pi$. On the contrary, 


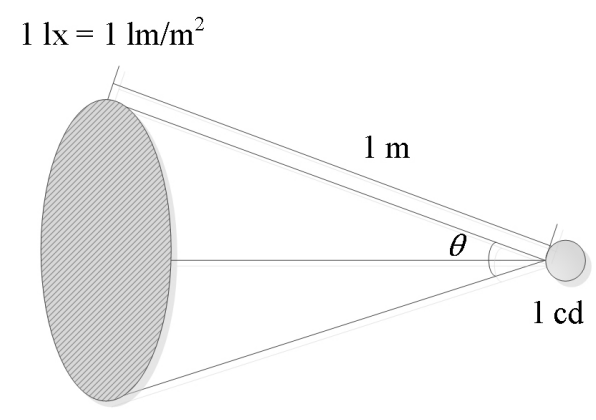

Fig. 4. Calculation of the luminous intensity.

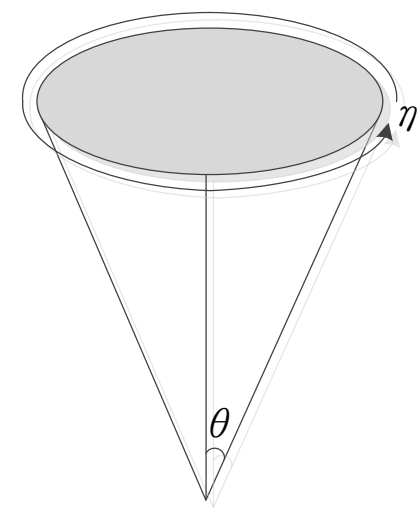

Fig. 5. Concept of the solid angle.

a solid angle of other light sources with regular angle is shown in Fig. 5 and is calculated using equation (5), where $\theta$ is $50 \%$ of the beam angle and $\eta$ is equal to $360^{\circ}$.

$$
\omega=\int_{\eta=0}^{2 \pi} \int_{\theta=0}^{\theta} \sin \theta \bullet d \theta \bullet d \eta
$$

For circular rotation with angle $\theta$, equation (6) is given by

$$
\omega=\int_{\theta=0}^{\theta} 2 \pi \bullet \sin \theta \bullet d \theta
$$

If equation (4) is substituted into equation (6), equation (7) is given by

$$
\phi=I \bullet 2 \pi(1-\cos \theta)[\operatorname{lm}]
$$

Finally, the luminous intensity $I$ is given by

$$
I=\frac{\phi}{2 \pi(1-\cos \theta)}[\mathrm{cd}]
$$

In equation (8), if the total luminous flux of a light source and the beam angle of $2 \theta$ are given, the center luminous intensity can be calculated [9]. In this paper, the performance of the searchlight was evaluated by calculating the center luminous intensity using equation (8).

$$
I=\frac{r^{2} \bullet E}{\cos \theta}[\mathrm{cd}]
$$

When the beam angle is not given, equation (9) is used to calculate the luminous intensity using the center illuminance. If $\theta$ is considered to be $0^{\circ}, I$ is calculated by multiplying the square of $r$ by $E$, where $I$ is the intensity, $E$ is the illuminance and $r$ is the measurement distance.

\subsection{Experimental configuration}

As a light source, a $9 \mathrm{~mm}$ in diameter and $95 \mathrm{~W}$ multi-chip package (MCP) was adopted to the system. Fig. 6 shows the MCP and the parabolic reflector of $620 \mathrm{~mm}$ in diameter, $110 \mathrm{~mm}$ in depth and $220 \mathrm{~mm}$ in focal distance.

(a)

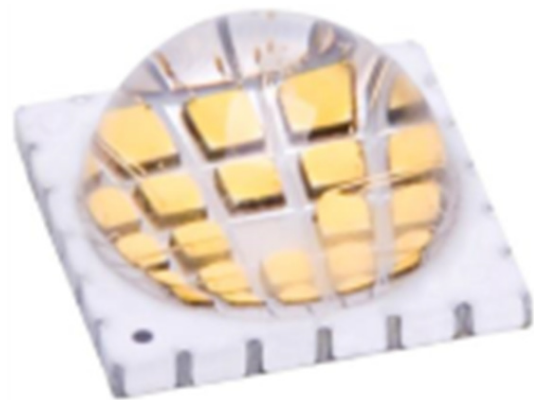

(b)

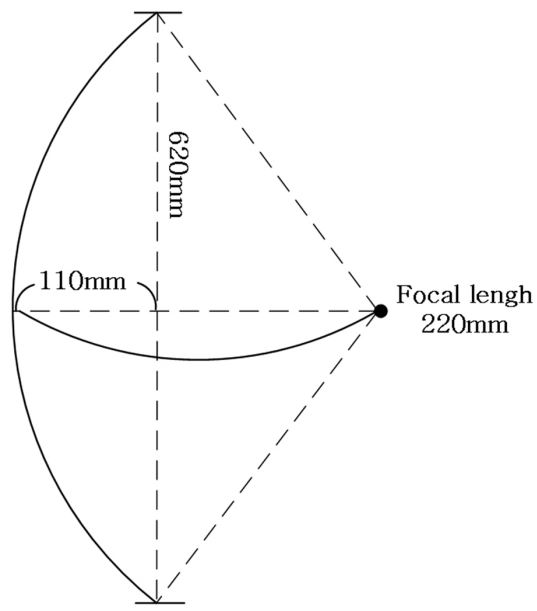

Fig. 6. A light source and a parabolic reflector. (a) MCP and (b) parabolic reflector.

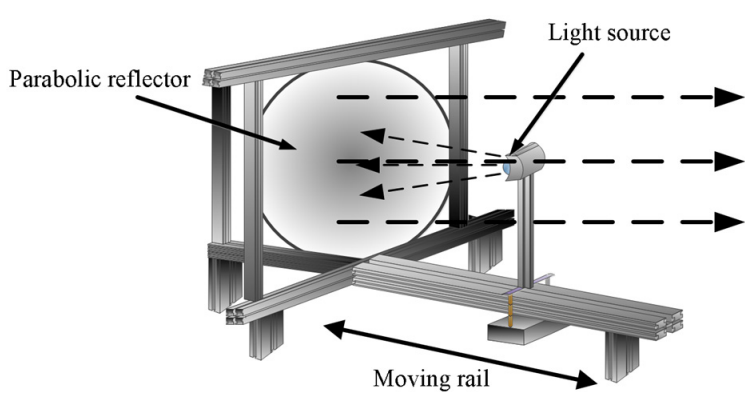

Fig. 7. Configuration of the experimental system. 
Figure 7 shows the configuration of the experimental system. The LED searchlight can collect light through the moving rails by changing the focal distance of the light source and fixing the parabolic reflector. The center height of the parabolic reflector and the MCP were designed to be $310 \mathrm{~mm}$. The water-cooling device was designed to improve the effect of heat dissipation because the high-power LED of $95 \mathrm{~W}$ was attached to the aluminum plate.

\section{EVALUATION AND ANALYSIS}

Figure 8 presents the ray-tracing simulation result with the designed light source and parabolic reflector. The simulation result confirmed that the rays were reflected in parallel [10].

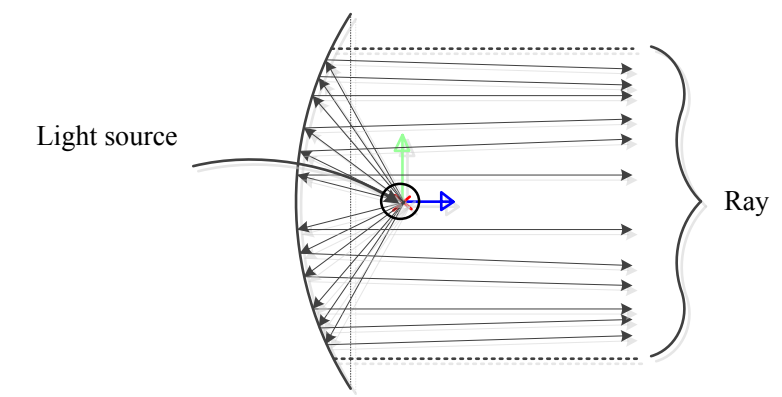

Fig. 8. Example of ray-tracing.

The maximum illuminance at the center was 40,110 lx in an area of $10 \mathrm{~m}^{2}$ and at a distance of $20 \mathrm{~m}$ from the light source as shown in Fig. 9 [11].

To obtain the beam angle and the luminous intensity at the center, an experiment was carried out as shown in Fig. 10. The searchlight was placed at the central $\mathrm{O}$ point and the maximum illuminance was measured at point A, which was located $20 \mathrm{~m}$ from the center. The distance $l$ was measured at point B, which has $10 \%$ of the illuminance of point $\mathrm{A}$, at a distance of $20 \mathrm{~m}$ from the central $\mathrm{O}$ point. At point $\mathrm{A}$, the maximum illuminance was $41,100 \mathrm{~lx}, l$ was $0.36 \mathrm{~m}$, and the beam angle $\theta$ computed using the Pythagoras theorem was $1.03^{\circ}$.

When the value of the beam angle $\theta$ is substituted into equation (8), equation (10) is given by

$$
I=\frac{7,800}{2 \pi(1-\cos \theta)}[\mathrm{cd}]
$$

The luminous intensity at the center, $I$, was approximately $7.7 \times 10^{6} \mathrm{~cd}$. The distance, $r$, is calculated using equation (9) when the luminance is $1 \mathrm{~lx}$.

$$
r=\sqrt{\frac{I}{E}}[\operatorname{lm}]
$$

Using equation (11), $r$ was $2.8 \mathrm{~km}$ at a luminance of $1 \mathrm{~lx}$. Table 1 shows the optical performance of the prototype LED searchlight at distances of $850 \mathrm{~m}, 1,000 \mathrm{~m}$, and 1,300 m.

Figure 11 shows a photograph of the experiment where the light was reflected to the top of a mountain, which was about 800

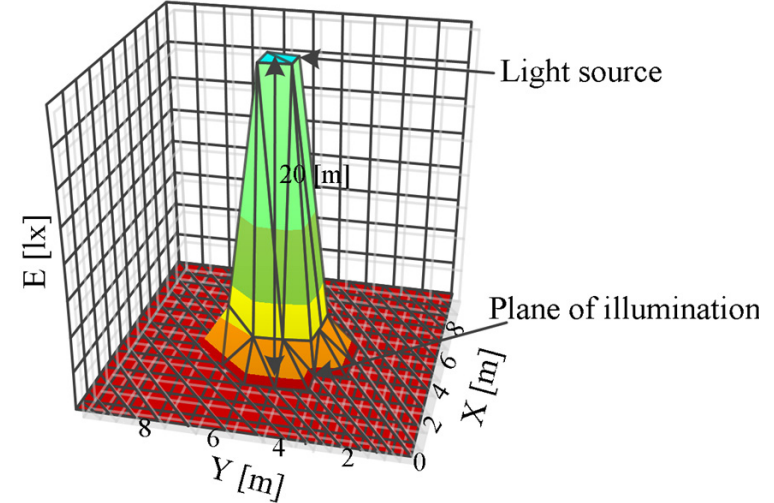

Fig. 9. Example of illuminance distribution.

(a)

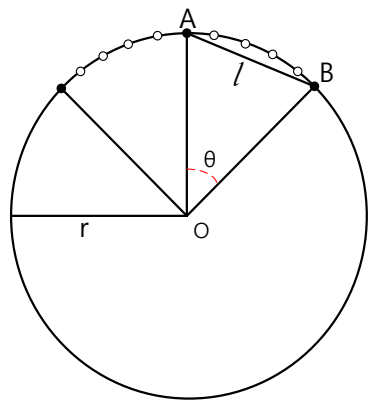

(b)

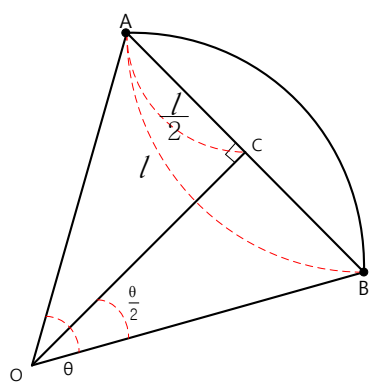

Fig. 10. Calculation diagram of the maximum illuminance. (a) Illuminance and (b) beam angle.

Table 1. Optical performance of the prototype LED searchlight.

\begin{tabular}{c||ccc}
\hline Distance $[\mathrm{m}]$ & 850 & 1,000 & 1,300 \\
\hline Center illuminance $[\mathrm{lx}]$ & 11.3 & 8.1 & 6.4 \\
\hline Luminous intensity at the center $\left[\mathrm{cd} \times 10^{6}\right]$ & 8.2 & 8.1 & 8.3 \\
\hline Distance at $1 \mathrm{~lx}[\mathrm{~km}]$ & 2.9 & 2.8 & 2.9 \\
\hline
\end{tabular}

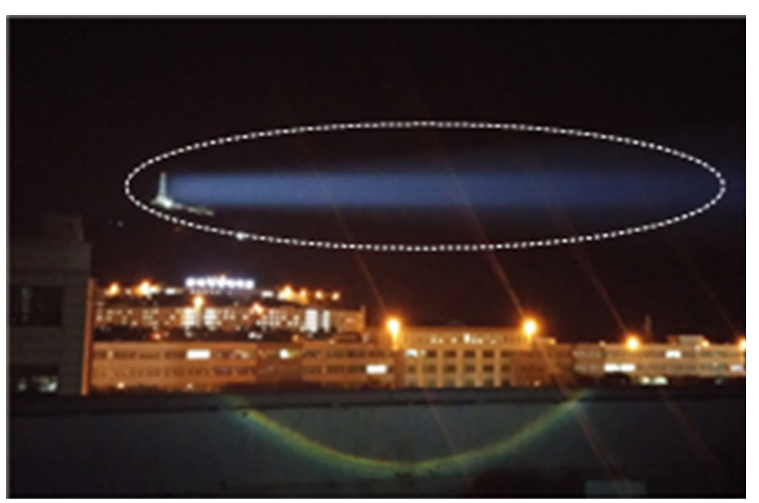

Fig. 11. Ray direction of LED searchlight. 
$m$ from the LED searchlight. As the beam angle of the searchlight calculated based on Fig. 10 reaches $1.03^{\circ}$, the light ray proceeds forward without any diffusion.

\section{CONCLUSIONS}

This paper described the development of a high condensing LED searchlight, using a parabolic reflector to replace a searchlight with a $1 \mathrm{~kW}$ xenon lamp. The light condensing system was composed of an LED package, a parabolic reflector, and a watercooling device. The luminous intensity at the center measured $20 \mathrm{~m}$ from the light source was $7.7 \times 10^{6} \mathrm{~cd}$. The distance between the light source and the point where the illumination intensity was $1 \mathrm{~lx}$ was calculated to be $2.8 \mathrm{~km}$.

The power consumption of the system was $95 \mathrm{~W}$, which is only $9.5 \%$ of the $1 \mathrm{~kW}$ xenon searchlight, and the beam angle was $1.03^{\circ}$. In a site experiment, it was confirmed that the light ray proceeds forward without any diffusion because of the narrow beam angle.

The diameter of the reflector is $620 \mathrm{~mm}$, but this could be reduced to $280 \mathrm{~mm}$ by modifying the radius of curvature, depending on the diameter and the beam angle of the LED package. The well designed LED searchlights have advantages including an instantaneous turn-on, a low power consumption, and a light weight.

\section{ACKNOWLEDGMENTS}

This research was supported by the National Research Foundation of Korea (NRF) grant funded by the Korean Government and the MSIP (Ministry of Science, ICT \& Future Planning), Korea, under the ITRC(Information Technology Research Center) support program supervised by the NIPA(National IT Industry
Promotion Agency) (NIPA-2014-H0301-14-1016).

\section{REFERENCES}

[1] S. J. Park and Y. L. Lee, Trans. Electr. Electron. Mater., 15, 201 (2014). [DOI: http://dx.doi.org/10.4313/TEEM.2014.15.4.201]

[2] E. D. Jung and Y. L. Lee, Trans. Electr. Electron. Mater., 15, 270 (2014). [DOI: http://dx.doi.org/10.4313/TEEM.2014.15.5.270]

[3] M. K. Kim and J. H. Park, The Current Trend and Industrial Strategies of Next LED, Proc. of KICS Winter Conference (Korean Institute of Communications and Information Sciences, 2010) p. 635-636.

[4] C. H. Tsuei, J. W. Pen and W. S. Sun, Opt Express, 16, 18692 (2008). [DOI: http://dx.doi.org/10.1364/OE.16.018692]

[5] E. R. Vidal, D. Otaduy, D. Ortiz, F. Gonzalez, F. Moreno, and J. M. Saiz, Optik, 125, 1657 (2014). [DOI: http://dx.doi.org/10.1016/ j.ijleo. 2013.09.064]

[6] R. Winston, J. C. Minano, and P. G. Benitez, Nonimaging Optics (Schools of Engineering \& Natural Science University of California, 2008) p. 24.

[7] L. Infante and S. Maci, IEEE Antennas Wirel Propag Lett., 2, 273 (2003). [DOI: http://dx.doi.org/10.1109/LAWP.2003.820685]

[8] D. Jenkins and R. Winston, J. Opt. Soc. Am., 13, 2106 (1996). [DOI: http://dx.doi.org/10.1364/JOSAA.13.002106]

[9] S. Zhao, K. Wang, F. Chen, and S. Liu, International Conference on Electronic Packaging Technology and High Density Packaging, 1491 (2012). [DOI: http://dx.doi.org/10.1109/ICEPTHDP.2012.6474889]

[10] C. H. Peng, X. N. Li, L. L. Xiong, P. Zhang, X. S. Liu, J. W. Wang, and X. S. Liu, Advances in Optoelectronics and Micro/ Nano-Optics, 1 (2012). [DOI: http://dx.doi.org/10.1109/ AOM.2010.5713510]

[11] G. G. Gregory, Modern Hightech (2014) p. 81. 